science. All of these were inspired by the earlier work of physicists and mathematicians, and often appealed to the Navier-Stokes equations. But they also introduced novel approximations and did not hesitate to use purely numerical methods when no analytical solutions were available. Meanwhile, the hydrodynamicists discovered that the real-life problems that the engineers were tackling involved deep questions in physics and mathematics. The study of the instability of fluid motions - the subject of Darrigol's fifth chapter - exemplifies these developments best: the two cultures began to meet, as the formulations of William Orr, Arnold Sommerfeld and Lord Rayleigh led both to the striking work of G. I. Taylor on cylindrical Couette flow and Werner Heisenberg on plane Poiseuille flow, and to the prescient work on boundary layers by Prandtl and Walter Tollmien.

Darrigol handles these developments with scholarship, insight and charm. One of the fascinating episodes he describes is the vortex theory of matter. For William Thomson (later Lord Kelvin) the idea that matter was made up of vortex rings had an extraordinary appeal: but the theory needed vortex rings to be stable, which unfortunately they were not. It seems to me that it was such failures, amid the striking success of James Maxwell's electromagnetic theory and other exciting developments in relativity and quantum mechanics, that were largely responsible for physicists' fading interest in fluid dynamics. It was thus largely left to the applied mathematicians and the engineers to fashion whatever successes the twentieth century could claim.

Darrigol takes us through these developments in the still incomplete history of the subject with a rare balance that accurately reflects its rich complexity (although I did miss mention of V. W. Ekman's friction layer in the rotating ocean and L. F. Richardson's heroic failure at numerical weather prediction). This is a book that all practising fluid dynamicists must read: I hope there will be a paperback edition soon, so that the strange history of the subject that Darrigol describes with such insight will become part of the intellectual legacy of interested students in engineering, mathematics and physics.

Roddam Narasimha is at the Jawaharlal

Nehru Centre for Advanced Scientific

Research, Jakkur, Bangalore 560 064, India.

\title{
Form and flow
}

\section{A sculptural approach to the heart.}

Philip J. Kilner

During my medical training and early years as a doctor, I was repeatedly drawn to art, even if itseemed to me then to lack relevance to medical work. Art felt liberating and satisfying, but subjective and impractical, whereas the kind of scientific thought that I wascoming to adopt seemed convincing and applicable, yet strangely out of touch with human feelings or values. I left medicine for several years and studied sculpture at Emerson College inSussex, England. As Martin Kemp has pointed out, science and art are nothomogeneous categories (Nature 434, 308-309; 2005). What I found atEmersonCollege was an observational approach tonatural science, and anartistic approach that was nourished by natural forms and processes without necessarily being representational. This was a rewarding mix, fostering discovery, creativity and a sense of wonder. The receptive, phenomenological approach we used owed much to Johann Wolfgang von Goethe (1749-1832), who regarded his scientific studies as more significant than his literary work.

Like Goethe, we made observations of form and transformation in plants, animal bones and the human skeleton. Wewere taught by John Wilkes, the originator of a range of elegantly sculpted surfaces called Flowforms, shaped to induce rhythmically alternating vortices inwater streaming over them

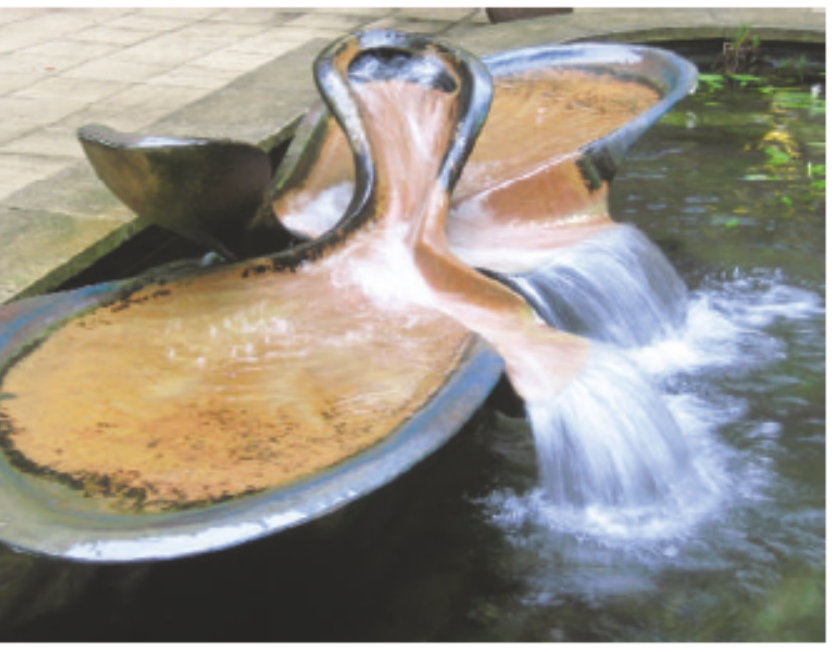

related to congenital heart disease and heart surgery.

But was art really significant as part of this mix, or was it just the practical approach that provided a basis for research on flow through the heart? The directengagement with three-dimensional form and flow was relevant, but other aspects of the artistic approach wereequally important.

One of these is the relative freedom to explore. Each artistic field offersits own playground for exploring the associations between phenomena. And the arts, traditionally at least, imply

(www.virbelaflowforms.anth.org.uk).We learned to guide the movements of water and shape the swirling, fluctuating vortices, gradually gaining insightinto the morphodynamics of flow. Creative engagement rarely resulted in a product that stood the test of time, but regularly led to fresh insight and discovery. I remember imagining and then shaping a variant of Wilkes' Flowform designto makea simple, asymmetric cavity that could convert continuous inflow to pulsatile outflow through a spontaneouscycle of accumulation and discharge. A pair of such forms (see above) can be seen at Royal Brompton Hospital in London.

Over the next 20 years, this discovery led me to explore the forms and dynamics of flow through heart cavities (see Nature 404, 759-761; 2000), to a career in cardiovascular imaging, and to research aesthetic appreciation. Beauty and, perhaps, meaning tend to be apparent not through analysis but throughinclusive, intuitive perception, which, like analytical and theoretical approaches, requires practice and training. Artistic exploration, whether among forms, colours or sounds, fosters a more flexible, inclusive awareness than analysis. If that kind of embracing awareness were adequately represented in scientific institutions as a counterpart to the analytical, I might feel less need towrite this. But as far as I have exerienced, the culture of science favours analysis at the expense of contextual awareness. The latter is at least as important, particularly whendealing with the organic, theernironmental, and the human. Philip J. Kilner is a consultant and reader in cardiovascular magnetic resonance at Royal Brompton Hospital and Imperial College, London, UK. 